\title{
An Intersectional Perspective on Access to HIV-Related Healthcare for Transgender Women
}

Ashley Lacombe-Duncan*

\begin{abstract}
Transgender women experience decreased access to HIV-related healthcare relative to cisgender people, in part due to pervasive transphobia in healthcare. This perspective describes intersectionality as a salient theoretical approach to understanding this disparity, moving beyond transphobia to explore how intersecting systems of oppression, including cisnormativity, sexism/transmisogyny, classism, racism, and HIV-related, gender nonconformity, substance use, and sex work stigma influence HIV-related healthcare access for transgender women living with HIV. This perspective concludes with a discussion of how intersectionality-informed studies can be enhanced through studying underexplored intersections and bringing attention to women's resiliency and empowerment.
\end{abstract}

Key words: access-to-care; health disparities; HIV/AIDS; intersectionality; transgender women

\section{Introduction}

Systemic exclusion of transgender women ${ }^{1}$ and particularly transgender women of color ${ }^{2}$ is associated with disproportionately high rates of HIV for this population. Access to HIV-related healthcare-including linkage to and retention in care, and initiation of and adherence to antiretroviral (ARV) treatments ${ }^{3}$-is of pivotal importance given that women living with HIV (WLWH) who are not retained in care may not initiate or adhere to ARV treatments, leading to virological failure and death. ${ }^{4}$

Among the small yet growing body of research that focuses on understanding HIV-related healthcare access for transgender WLWH, ${ }^{5-16}$ quantitative studies suggest that transgender WLWH experience differential access, ${ }^{7,12,16}$ including lower retention in care, ${ }^{16}$ current ARV use, ${ }^{7}$ and ARV adherence. ${ }^{12}$ Although most quantitative analyses of HIV among transgender women do not explicitly apply theory, several qualitative studies have employed an intersectional approach to understanding the experiences of transgender WLWH. ${ }^{5,6,13}$
HIV-related healthcare access disparities

for transgender women: intersectionality

Intersectionality is a critical social theory that allows for an understanding of how multiple social identities (e.g., HIV status and gender identity) intersect at the microlevel of transgender WLWH's individual experiences to enact systems of privilege and oppression (e.g., HIV-related stigma and cisnormativity) operating at the macrolevel of society (see Table 1, key definitions). ${ }^{17-19}$ Studies employing an intersectional approach to understanding HIV-related healthcare access for transgender WLWH engage with themes related to macrolevel oppression (e.g., HIV-related stigma and cisnormativity), how these concepts relate to the organization of care (e.g., exclusion of transgender WLWH's needs from healthcare research), and how this translates into the everyday experience of transgender WLWH in healthcare settings (e.g., stigmatizing care).

Beyond those explicitly applying intersectionality, qualitative studies of access to HIV-related healthcare for transgender WLWH highlight experiences of transphobic stigma and discrimination within and across

Factor-Inwentash Faculty of Social Work, University of Toronto, Toronto, Ontario, Canada.

*Address correspondence to: Ashley Lacombe-Duncan, MSW, Factor-Inwentash Faculty of Social Work, University of Toronto, 246 Bloor Street West, Toronto, Ontario, Canada, M5S 1V4, E-mail: ashley.lacombe.duncan@utoronto.ca

(c) Ashley Lacombe-Duncan 2016; Published by Mary Ann Liebert, Inc. This Open Access article is distributed under the terms of the Creative Commons License (http://creativecommons.org/licenses/by/4.0), which permits unrestricted use, distribution, and reproduction in any medium, provided the original work is properly credited. 
Table 1. Intersectionality and Transgender Women Living with HIV: Key Definitions

\begin{tabular}{|c|c|}
\hline Term & Definition \\
\hline Oppression & The systematic and ongoing denial of one group's access to the resources of society by another group (p. 6). ${ }^{17}$ \\
\hline Internalized HIV-related stigma & $\begin{array}{l}\text { The degree to which people living with HIV endorse negative beliefs and feelings driven by society about } \\
\text { themselves (p. 1163). }\end{array}$ \\
\hline Enacted HIV-related stigma & Subtle or overt forms of discrimination as a result of one's HIV status (p. 1163). ${ }^{21}$ \\
\hline Anticipated HIV-related stigma & $\begin{array}{l}\text { The degree to which people living with HIV believe they will experience prejudice and discrimination from } \\
\text { others in the future (p. 1163). }{ }^{21}\end{array}$ \\
\hline Cisnormativity & $\begin{array}{l}\text { The sociocultural assumptions and expectations that all people are cis-sexual and/or have a cisgender body } \\
\text { (p. 356). } .^{33}\end{array}$ \\
\hline Sexism & The belief in the inherent superiority of one sex and thereby the right to dominance (p. 45$){ }^{19}$ \\
\hline Transmisogyny & $\begin{array}{l}\text { The ridiculing or dismissal of a transgender person not merely for failing to live up to gender norms, but for their } \\
\text { expressions of femaleness or femininity (p. 14). }{ }^{28}\end{array}$ \\
\hline Gender nonconformity stigma & $\begin{array}{l}\text { Stigma or discrimination of cisgender and transgender women who have a masculine/masculine-of-center } \\
\text { gender presentation. }\end{array}$ \\
\hline Racism & The belief in the inherent superiority of one race and thereby the right to dominance (p. 45$).{ }^{19}$ \\
\hline
\end{tabular}

healthcare settings for transgender WLWH as a particularly salient barrier to HIV-related healthcare access. ${ }^{5,6,8,10,11,13,15}$ Scholars have suggested that prioritizing one category of identity over others does not fully consider the context of inequity in which poor health outcomes proliferate. Thus, these studies also describe how access to HIV-related healthcare is differentially experienced at the intersection of multiple forms of stigma beyond transphobia, including, for example, HIV-related and gender nonconformity stigma.

An intersectional perspective has the potential to enhance our understanding of processes of social exclusion operating across several intersecting oppressions, ultimately informing how we understand HIV-related healthcare access disparities for transgender women. This perspective reviews the literature about access to HIVrelated healthcare for transgender WLWH through an intersectional lens. Next, this perspective discusses how intersectionality-informed studies can be enhanced through studying underexplored intersections and bringing attention to women's resiliency and empowerment.

\section{Applying Intersectionality to Understand Access} to HIV-Related Healthcare for Transgender Women Most research that explores HIV-related healthcare has looked at the experiences of transgender WLWH grouped with women, ${ }^{5}$ LGBT people broadly, ${ }^{6}$ or men who have sex with men. ${ }^{20}$ When studies do focus specifically on transgender women or deaggregate data by transgender identity, the voices of transgender WLWH show a complexity of experiences based on intersecting systems of oppression, including HIV-related stigma, cisnormativity, sexism/transmisogyny, gender nonconformity stigma, classism, sex work, and substance use stigma, and racism (Fig. 1).
HIV-related stigma

HIV-related stigma is a well-documented barrier to HIV prevention, treatment, care, and support for all people living with HIV. ${ }^{21}$ The impact of HIV-related stigma at a systemic level is evident in research about transgender WLWH, whereby the vast majority of the limited number of studies about transgender women and HIV seek to identify, label, quantify, and reduce HIV risk, while there has been little focus on their treatment, care, and support needs postdiagnosis. ${ }^{22}$ This observation is consistent with broader knowledge production about HIV and women, which tends also to focus on prevention. ${ }^{23}$

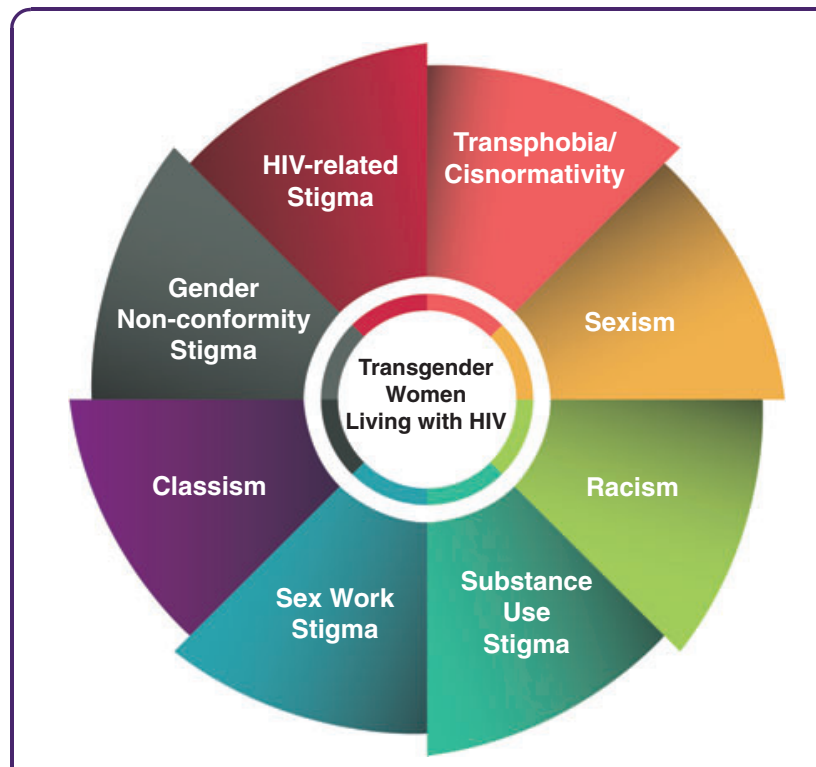

FIG. 1. Intersecting stigmas influencing access to HIV-related healthcare for transgender women. 
Although prevention is essential, this focus on women as "risky" may perpetuate long-held societal stereotypes of WLWH as "diseased" or "dirty," ${ }^{24}$ and is particularly detrimental toward transgender WLWH who may be subject to additional assumptions (e.g., presumption of sex work) by their healthcare providers. ${ }^{15}$ At an individual level, internalized HIV-related stigma is associated with reduced self-esteem and self-worth, and increased depression or suicidality among cisgender and transgender $\mathrm{WLWH},{ }^{5}$ which also influence access. Both internalized and anticipated HIV-related stigma are associated with decreased engagement in HIV-related care among cisgender and transgender WLWH. ${ }^{25}$

\section{Cisnormativity}

On a systemic level, cisnormativity contributes to an underinvestigation of HIV-related care needs for transgender women, such as a lack of information on the interaction between hormones and HIV medications. ${ }^{6,10,13}$ Physician support of women's continuing their hormones is a facilitator of initiating ARV treatment. ${ }^{8}$ Thus, the exclusion of transgender-specific HIV research poses barriers to HIV-related healthcare access at an individual level for transgender women. Studies also consistently suggest that transgender WLWH report fewer positive interactions with their healthcare providers than cisgender WLWH. ${ }^{12}$

Similarly to HIV-related stigma, anticipated and enacted transphobia may prevent women from disclosing their transgender identity to healthcare providerslimiting their access to gender-affirming care-and may result in avoidance of healthcare altogether. ${ }^{26,27}$

\section{Sexism/transmisogyny}

Serano $^{28}$ describes the intersection between transphobia and sexism as transmisogyny. Transmisogyny has been underexplored in the academic literature to date, and has predominantly been applied in the field of feminist studies to explain the exclusion of transgender women from women's spaces ${ }^{29}$ or to describe interlocking forms of gender oppression. ${ }^{30}$ However, several important links between transmisogyny and barriers to access to care can be made, such as the potential impact of transmisogyny on women's self-esteem or women's safety. For example, transmisogyny may help to explain increased violence against transgender women who do sex work compared with cisgender women. ${ }^{11}$

\section{Gender nonconformity stigma}

Healthcare provider insensitivity, hostility, and ignorance of transgender health are associated with not "passing" as one's identified gender. ${ }^{27}$ Despite the potential importance of gender conformity in promoting HIV-related healthcare access, gender-affirming surgery may be more difficult to access for transgender WLWH than transgender women not living with HIV. ${ }^{10}$ Lastly, gender nonconforming or "not passing" transgender WLWH report increased violence ${ }^{15}$; thus, access to gender-affirming healthcare may play a pivotal role in women's safety and, ultimately, in their ability to access to HIV-related healthcare.

\section{Classism}

Gender nonconformity stigma is often a class-based issue for transgender WLWH-without health insurance, women may not be able to afford gender-affirming care, cannot express their gender, and experience increased risk of violence or discrimination. ${ }^{8,15}$ Moreover, among transgender people, economic discrimination itself is a strong predictor of experiencing violence. ${ }^{31}$ Although poverty is a large issue among WLWH generally, transgender WLWH are more likely than cisgender people living with HIV to experience socioeconomic marginalization on multiple fronts, such as lower income, homelessness, and lack of health insurance, ${ }^{7,9}$ posing a socioeconomic disadvantage to HIV-related healthcare access.

\section{Sex work and substance use stigma}

Sex work stigma, HIV-related stigma, and drug use stigma intersect to limit women's access to HIV-related care. ${ }^{5,11}$ Transgender WLWH are more likely to have a good relationship with their primary care physician if not using substances or involved in sex work. ${ }^{11}$ These intersections are found to contribute to avoidance of care, and subsequent injection drug-related complications such as hepatitis $\mathrm{C}$ among transgender WLWH. ${ }^{11}$

\section{Racism}

Few studies have specifically looked at the impact of racism on access to HIV-related care for transgender WLWH. It has been suggested through qualitative work that transgender WLWH of color report a greater sense of fatalism around HIV than white women, ${ }^{13}$ whereas another qualitative study found that racism permeated both individual interactions with healthcare providers and systemic exclusion from research and healthcare planning. ${ }^{5}$ These findings can be contextualized within research among transgender people broadly, which suggests the intersection between cisnormativity/transphobia and systemic racism in producing differential experiences with respect 
to healthcare access for transgender people of color, such as access to a physician. ${ }^{32}$

\section{Intersectional Considerations Moving Forward}

As demonstrated, an intersectional analysis brings to light the experiences of transgender women situated within multiple intersecting oppressions. Future intersectionality-informed studies can be enhanced by focusing on underexplored intersections and highlighting women's resiliency and empowerment in HIVrelated healthcare navigation.

\section{Underexplored intersections}

Few studies examine the HIV-related healthcare experiences of sexual minority transgender WLWH-women who have sex with other women or identify as lesbian, bisexual, gay, or queer. In addition, no identifiable studies discuss the experiences of transgender WLWH with disabilities. Migration and immigration status may also influence women's access to care, yet is often overlooked in studies of transgender WLWH. It is likely that transgender WLWH who have immigrated recently or are refugees have limited access not only to HIV-related healthcare but also to gender-affirming care, a major facilitator of HIV-related healthcare access. Futures studies conducted from an intersectional lens could aim to include sexual minority, differently-abled, and immigrant transgender WLWH.

\section{Resilience and empowerment}

Much research depicts transgender WLWH as a uniformly disadvantaged group where women must struggle to just survive intersecting stigmas. For example, when studies talk about coping, they often talk about "poor" coping, ${ }^{10}$ or the use of substances to cope with stigma. ${ }^{13}$ This depiction overlooks women's adaptive coping mechanisms, resilience, and empowerment. Notably, Logie et al. ${ }^{5}$ discuss women's resilience, resistance to internalized stigma, and self-determination. The authors also highlight how women cope through faith and prayer, joining support groups, and challenging stigma through advocating, political mobilization, and education. ${ }^{5}$ Political mobilization is particularly salient to transgender populations. Barriers and facilitators to healthcare access are often noted as in the hands of healthcare providers or those who organize healthcare. Future studies could further explore adaptive coping mechanisms and highlight women's resiliency and document involvement of transgender WLWH in activism, as well as how this involvement contributes to intrapersonal (e.g., mental well-being) and interpersonal (e.g., HIV-related healthcare provider interaction) factors.

\section{Conclusions}

HIV is a social epidemic that disproportionately affects people experiencing social exclusion across multiple domains of life and based on multiple facets of identity. By applying an intersectional approach to research about HIV-related healthcare access for transgender WLWH, we may develop ways to reduce intersectional stigmatization, build on strengths, increase access, and, ultimately, improve the health and well-being of an over-represented and underresearched population.

\section{Acknowledgments}

This work was funded by a Lupina Junior Doctoral Fellowship with the Comparative Program on Health and Society at the University of Toronto's Munk School of Global Affairs. I gratefully acknowledge the feedback of Dr. Peter A. Newman and Dr. Carmen H. Logie as well as the CPHS fellows, director, and coordinator on the work from which this article evolved.

\section{Author Disclosure Statement}

No competing financial interests exist.

\section{References}

1. Baral SD, Poteat T, Strömdahl S, et al. Worldwide burden of HIV in transgender women: a systematic review and meta-analysis. Lancet Infect Dis. 2013;13:214-222.

2. Herbst JH, Jacobs ED, Finlayson TJ, et al. Estimating HIV prevalence and risk behaviors of transgender persons in the United States: a systematic review. AIDS Behav. 2008;12:1-17.

3. Mugavero MJ, Amico KR, Horn T, Thompson MA. The state of engagement in HIV care in the United States: from cascade to continuum to control. Clin Infect Dis. 2013;57:1164-1171.

4. Ulett KB, Willig JH, Lin HY, et al. The therapeutic implications of timely linkage and early retention in HIV care. AIDS Patient Care STDS. 2009;23:41-49.

5. Logie $\mathrm{CH}$, James $\mathrm{L}$, Tharao W, Loutfy MR. HIV, gender, race, sexual orientation, and sex work: a qualitative study of intersectional stigma experienced by HIV-positive women in Ontario, Canada. PLoS Med. 2011;8:e1001124.

6. Logie $\mathrm{CH}$, James L, Tharao W, Loutfy MR. "We don't exist": a qualitative study of marginalization experienced by HIV-positive lesbian, bisexual, queer and transgender women in Toronto, Canada. J Int AIDS Soc. 2012;15:17392.

7. Melendez RM, Exner TA, Ehrhardt AA, Dodge B, et al. Health and health care among male-to-female transgender persons who are HIV positive. Am J Public Health. 2006;96:1034-1037.

8. Melendez RM, Pinto RM. HIV prevention and primary care for transgender women in a community-based clinic. J Assoc Nurses AIDS Care. 2009:20:387-397.

9. Mizuno Y, Frazier EL, Huang P, Skarbinski J. Characteristics of transgender women living with HIV receiving medical care in the United States. LGBT Health. 2015;2:228-234.

10. Schilder A, Laframboise $S$, Hogg R, et al. "They don't see our feelings." The health care experiences of HIV-positive transgendered persons. J Gay Lesbian Med Assoc. 1998;2:103-111. 
11. Schilder AJ, Kennedy C, Goldstone IL, et al. "Being Dealt With as a Whole Person." Care seeking and adherence: the benefits of culturally competent care. Soc Sci Med. 2001;52:1643-1659.

12. Sevelius JM, Carrico A, Johnson MO. Antiretroviral therapy adherence among transgender women living with HIV. J Assoc Nurses AIDS Care. 2010;21:256-264.

13. Sevelius JM, Patouhas E, Keatley JG, Johnson MO. Barriers and facilitators to engagement and retention in care among transgender women living with human immunodeficiency virus. Ann Behav Med. 2014;47:5-16.

14. Sevelius JM, Saberi P, Johnson MO. Correlates of antiretroviral adherence and viral load among transgender women living with HIV. AIDS Care. 2014;26:976-982.

15. Wilson EC, Arayasirikul S, Johnson K. Access to HIV care and support services for African American transwomen living with HIV. Int J Transgend. 2013;14:182-195.

16. Yehia BR, Fleishman JA, Moore RD, Gebo KA. Retention in care and health outcomes of transgender persons living with HIV. Clin Infect Dis. 2013;57:774-776.

17. Hill Collins P. Black Feminist Thought Knowledge, Consciousness, and the Politics of Empowerment. New York: Routledge, 2000.

18. Bowleg $\mathrm{L}$. The problem with the phrase women and minorities: intersectionality-an important theoretical framework for public health. Am J Public Health. 2012;102:1267-1273.

19. Lorde A. Sister Outsider. New York: Crossing Press, 1984.

20. Tanner AE, Reboussin BA, Mann $L$, et al. Factors influencing health care access perceptions and care-seeking behaviors of immigrant Latino sexual minority men and transgender individuals: baseline findings from the HOLA intervention study. J Health Care Poor Underserved. 2014;25:1679-1697.

21. Earnshaw VA, Chaudoir SR. From conceptualizing to measuring HIV stigma: a review of HIV stigma mechanism measures. AIDS Behav. 2009;13:1160-1177.

22. Sevelius JM, Keatley J, Gutierrez-Mock L. HIV/AIDS programming in the United States: considerations affecting transgender women and girls. Womens Health Issues. 2011;21:S278-S282.

23. Lunny C, Shearer BD, Cruikshank J, et al. Women in HIV conference research: trends and content analysis of abstracts presented at $17 \mathrm{HIV/AIDS}$ conferences from 2003 to 2010. Womens Health Issues. 2011;21:407-417.

24. Lawless S, Kippax S, Crawford J. Dirty, diseased and undeserving: the positioning of HIV positive women. Soc Sci Med. 1996;43:1371-1377.
25. Christopoulos KA, Massey AD, Lopez AM, et al. "Taking a half day at a time:" patient perspectives and the HIV engagement in care continuum. AIDS Patient Care STDs. 2013;27:223-230.

26. Cruz TM. Assessing access to care for transgender and gender nonconforming people: a consideration of diversity in combating discrimination. Soc Sci Med. 2014;110:65-73.

27. Xavier J, Bradford J, Hendricks M, et al. Transgender health care access in Virginia: a qualitative study. Int J Transgenderism. 2013;14:3-17.

28. Serano J. Whipping Girl: A Transsexual Woman on Sexism and the Scapegoating of Femininity. Berkeley, CA: Seal Press, 2007.

29. Weber S. "Womanhood does not reside in documentation": queer and feminist student activism for transgender women's inclusion at women's colleges. J Lesbian Stud. 2016;20:29-45.

30. Fu M. What will it take to end gender-based violence? Womens Stud J. 2015;29:50-59.

31. Lombardi EL, Wilchins RA, Priesing D, Malouf D. Gender violence: transgender experiences with violence and discrimination. J Homosex. 2001;42:89-101.

32. Kenagy GP. Transgender health: findings from two needs assessment studies in Philadelphia. Health Soc Work. 2005;30:19-26.

33. Bauer GR, Hammond R, Travers R, et al. "I don't think this is theoretical; This is our lives": How erasure impacts health care for transgender people. J Assoc Nurses AIDS Care. 2009;20:348-361.

34. Gordon AR, Meyer IH. Gender nonconformity as a target of prejudice, discrimination, and violence against LGB individuals. J LGBT Health Res. 2007;3:55-71.

Cite this article as: Lacombe-Duncan A (2016) An intersectiona perspective on access to HIV-related healthcare for transgender women, Transgender Health 1:1, 137-141, DOI: 10.1089/trgh.2016.0018.

\section{Abbreviations Used}

$\mathrm{ARV}=$ antiretroviral

WLWH $=$ women living with HIV

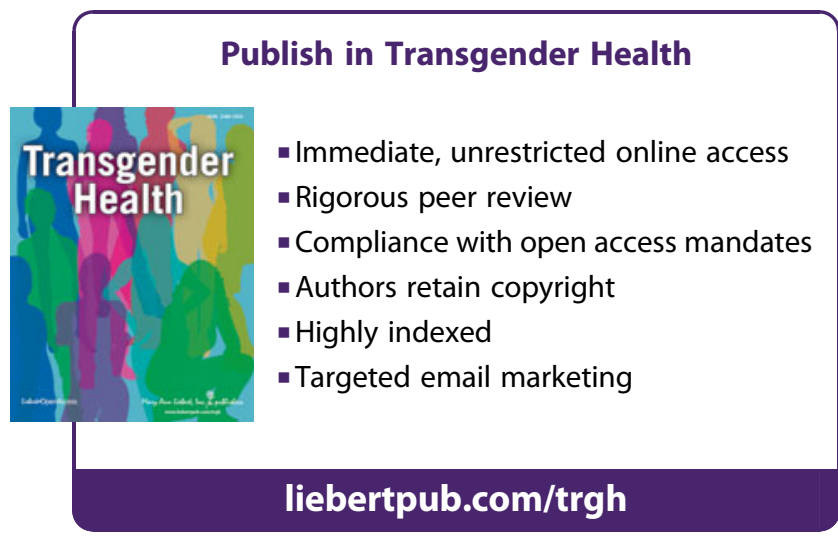

